\title{
Coulisses
}

Revue de théâtre

\section{Les « didascalies implicites » : l'exemple de Molière}

\section{Elsa Tadier}

\section{OpenEdition}

\section{Journals}

Édition électronique

URL : https://journals.openedition.org/coulisses/966

DOI : $10.4000 /$ coulisses. 966

ISSN : 2546-9460

\section{Éditeur}

Presses universitaires de Franche-Comté

\section{Édition imprimée}

Date de publication : 31 décembre 2009

Pagination : 43-54

ISBN : 978-2-84867-270-0

ISSN : 1150-594X

\section{Référence électronique}

Elsa Tadier, "Les « didascalies implicites » : I'exemple de Molière », Coulisses [En ligne], 39 | Automne 2009, mis en ligne le 30 novembre 2016, consulté le 29 décembre 2022. URL : http:// journals.openedition.org/coulisses/966; DOI : https://doi.org/10.4000/coulisses.966

Ce document a été généré automatiquement le 29 décembre 2022.

Tous droits réservés 


\title{
Les « didascalies implicites » : l'exemple de Molière
}

\author{
Elsa Tadier
}

1 En homme de scène, Molière, conscient du danger que représente pour le théâtre la publication d'une pièce, avertit ses lecteurs dans l'avis de L'Amour médecin :

[...] on sait bien que les comédies ne sont faites que pour être jouées, et je ne conseille de lire celle-ci qu'aux personnes qui ont des yeux pour découvrir dans la lecture tout le jeu du théâtre ${ }^{1}$.

2 Cet avertissement constituera notre point de départ. Il nous invite à formuler l'hypothèse que la scénographie des pièces de Molière ne peut être élaborée seulement à partir des didascalies lacunaires qui jalonnent le texte comme autant d'incises situées « hors jeu », mais aussi et surtout à partir des indications implicitement fournies tout au long de son développement par le rôle des personnages qu'il met en scène, par le texte vif de leur rôle. C'est le domaine, plus ou moins déchiffrable, de la didascalie implicite. Cette notion rejoint avec quelques nuances celle de "didascalie interne" proposée par Anne Ubersfeld, pour qualifier les indications de jeu intégrées à la parole des personnages, ou «tous les éléments [...] dans le dialogue qui ont une fonction de commande de la représentation ${ }^{2}$ », la notion de didascalie implicite insistant plus, pour sa part, sur le potentiel suggestif, la lisibilité et la fugacité des mots et procédés qui, dans l'écriture, peuvent orienter la représentation scénique.

Dans le théâtre de Molière, ces indications telles que les définit Anne Ubersfeld, droit issues du texte prononcé par les comédiens, nous apparaissent d'autant plus essentielles que les didascalies explicites y sont peu nombreuses. Nous imputerons leur rareté à deux motifs majeurs: d'une part la défiance du théâtre classique envers ce procédé d'écriture qui sera nommé plus tard dans l'histoire du théâtre "didascalie »; d'autre part le triple statut de Molière : celui de dramaturge, mais aussi de comédien principal et de "metteur en scène" de ses propres pièces, qui rendent moins nécessaire la présence de didascalies. Dès lors que, comme on va le voir, le procédé se généralise chez lui jusqu'à devenir un principe d'écriture théâtrale, la question posée par son analyse se renverse en celle-ci : comment la parole, sous la plume de Molière, 
devient-elle porteuse de jeu? Car le dramaturge, qui connait comme d'intuition les ressources du langage, réussit à établir dans ses comédies toute une scénographie sousjacente et fort utile pour qui tentera de relever le défi lancé par Molière de « découvrir dans la lecture tout le jeu de théâtre ».

\section{La mise en contexte du texte}

\section{Le décor}

4 Chez Molière, rares sont les didascalies implicites au sujet du décor, telles que la description par Horace de la maison qui renferme Agnès : « Un jeune objet qui loge en ce logis/Dont vous voyez ici que les murs sont rougis ${ }^{3} »$. Ici, l'indication est claire : le décor principal représente une maison aux murs rouges. Mais souvent, on est amené à supposer le lieu où se déroule l'action. Dans nombre de pièces, notamment, et comme ici pour L'Ecole des Femmes, la rue est le décor idéal pour le type de rencontres qu'impose le dramaturge à ses personnages. Supposition que nous confirment lorsqu'elles existent - les didascalies. D'ailleurs, traditionnellement, le décor de la scène comique est celui d'un carrefour, dans une ville, comme on le voit souvent chez Molière.

5 Toutefois, toutes les pièces de Molière n'ont pas pour décor un cadre extérieur. Les paroles des personnages nous suggèrent parfois qu'ils se trouvent dans un salon - décor mondain par excellence - comme c'est le cas dans Les Précieuses ridicules où Cathos et Magdelon miment les usages de la société mondaine. De manière générale, les décors intérieurs sont relativement fréquents, ainsi que nous l'indiquent les références aux meubles ou aux pièces comme le petit cabinet dans Le Tartuffe. Tout en étant variées, les didascalies implicites de décor, chez Molière, se font donc relativement rares et peu détaillées.

\section{Les costumes}

6 Pour les costumes, notre constat est le même : nous ne possédons que peu d'indications implicites. Néanmoins, il arrive que le texte de Molière se fasse très précis. L'Ecole des maris en est un bon témoignage. Il s'agit de la tirade de Sganarelle, qui reproche son accoutrement - auquel il oppose le sien propre - à son frère Ariste :

Ne voudriez-vous point, dis-je, sur ces matières,

De vos jeunes muguets m'inspirer les manières?

M'obliger à porter de ces petits chapeaux

Qui laissent éventer leurs débiles cerveaux, [...]

De ces petits pourpoints sous les bras se perdant,

Et de ces grands collets jusqu'au nombril pendant?

De ces manches qu'à table on voit tâter les sauces,

Et de ces cotillons appelés hauts-de-chausses?

De ces souliers mignons, de rubans revêtus

Qui vous font ressembler à des pigeons pattus?

Et de ces grands canons où, comme des entraves,

On met tous les matins ses deux jambes esclaves,

Et par qui nous voyons ces messieurs les galants

Marcher écarquillés ainsi que des volants? 
Je vous plairais, sans doute, équipé de la sorte ;

Et je vous vois porter les sottises qu'on porte ${ }^{4}$.

Dans ce cas, le texte pousse la précision jusqu'aux détails (la longueur des collets par exemple ou encore celle des manches). Nous pouvons également songer à la tirade d'Alceste qui condamne - en recourant aux mêmes procédés que Sganarelle l'excentricité du costume du marquis dans Le Misanthrope. De même, dans la dernière scène de cette même pièce, nous pouvons penser à la fameuse désignation d'Alceste par Célimène, qui le décrit comme "l'homme aux rubans verts " dans un billet destiné à un autre que lui. Cette désignation oriente ainsi la représentation que le lecteur se fait du personnage d'Alceste par la mention des rubans et de leur couleur. Il existe d'autres indications, moins riches, à propos du costume des personnages dans les comédies de Molière, mais rappelons combien elles se font rares et combien celles que nous possédons peuvent apparaitre précieuses au lecteur.

\section{La mise en jeu du texte}

Les suggestions scéniques du texte de Molière peuvent révéler un jeu tout en nuances. Le classement que nous avons construit s'appuiera en partie sur les catégories établies d'une part par Sabine Chaouche dans L'Art du comédien, et d'autre part par Gabriel Conesa dans Le Dialogue moliéresque.

\section{La position du corps sur scène}

\section{- Les positions insolites}

Lorsque certaines situations amènent les personnages à se mettre dans une position inhabituelle, le texte de Molière le mentionne clairement. Ce sont les positions que Sabine Chaouche nomme « insolites » parce qu'elles « illustrent des défauts stigmatisés par l'art oratoire qui rejette toute autre position que celle de se tenir debout ${ }^{6} »$. Souvenons-nous ainsi de la célèbre scène des Fourberies de Scapin où Géronte s'enferme dans un sac, sur la suggestion de Scapin. Cette position, considérée comme basse dans le théâtre classique, surprend d'autant plus qu'elle est révélatrice d'une inversion des rapports entre maître et valet. Autrement dit, le consentement de Géronte à adopter cette posture le ridiculise largement.

Dans d'autres cas, comme Le Tartuffe, il s'agira, pour le personnage de se cacher sous la table. Pensons également à la scène de danse où le maître de monsieur Jourdain n'a de cesse de rectifier sa posture. Il s'agit d'abord de la position de sa « jambe droite », puis de ses «épaules» ou sa "pointe $d[e]$ pied $^{7}$ ». Ici, la posture du personnage est hautement comique, et la scène dépeint un tableau fort peu noble.

\section{- Les positions civiles}

11 La position civile par excellence, chez Molière, est la position assise - fréquente car usuelle dans la société mondaine. Nous pouvons citer les exemples suivants, tirés des paroles des personnages :

1. Un siège au frais ici [...]

2. Allons, des sièges. Holà ! laquais, laquais, laquais.

3. Allons, vite, ma chaise, et des sièges à tout le monde ${ }^{8}$. 
Dans chacun de ces cas, l'indication implicite préfère en passer par la désignation d'un élément du décor - le siège - plutôt que de faire dire à un des protagonistes qu'il va s'asseoir. On atteint donc ici un second degré d'implicite où l'indication est lisible, mais suggère l'idée que la parole moliéresque est stratifiée. L'essentiel de la visualisation du jeu ne pourra donc être opéré que par un travail du lecteur sur le texte. On remarquera par ailleurs que les positions civiles sont révélatrices des codes sociaux : le noble Dom Juan nous le suggère, qui, pour éviter un remboursement de dettes, flatte son créancier (simple petit bourgeois) en le traitant symboliquement d'égal à égal puisqu'il ordonne à ses domestiques de remplacer le pliant qu'on lui assigné par un fauteuil du même type que le sien. Ainsi, la civilité trahit une hiérarchie sociale complexe où l'inférieur doit conserver sa position par la qualité du siège sur lequel il prend place.

\section{- La localisation dans l'espace}

13 Souvent, la position d'un personnage sur scène demeure dépendante de repères, euxmêmes scéniques, comme l'objet ou le protagoniste par rapport auxquels il est placé. Ainsi, tantôt la localisation d'Orgon sera définie par rapport à la table, tantôt celle de la Comtesse d'Escarbagnas est déterminée par celle de son siège. Ces rapports dans l'espace mettent en évidence l'interférence entre comédiens et décor. Cela est flagrant dans un exemple comme celui des Précieuses ridicules, où la localisation de Mascarille se fait sur un mode plus implicite encore :

Quoi? Toutes deux contre mon cœur, en même temps! m'attaquer à droite et à

gauche ! Ah! c'est contre le droit des gens [...] $]^{9}$.

Cette exclamation nous renseigne sur la place de Mascarille, à savoir entre Cathos et Magdelon. Dans un certain nombre d'autres pièces, Molière recourt au même procédé. On voit donc que ce n'est plus seulement la parole qui entraîne une action mais parfois l'action scénique qui alimente la parole, dans une dynamique d'influences réciproques essentielle à l'efficacité et à la cohérence du texte.

\section{Les déplacements scéniques}

\section{- Les entrées et sorties}

15 En général, chez Molière, l'ouverture ou la fermeture d'une scène se manifeste par l'entrée ou bien la sortie d'un personnage. Il s'agit de faire annoncer par un protagoniste déjà en scène l'arrivée d'un autre, ou son départ : cela permet de préparer la scène suivante, sans que le changement apparaisse trop artificiel. On le constate dans de nombreux cas : par exemple dans Le Médecin volant, où la première scène se clôt sur l'arrivée de Sganarelle, que recherche son maître: «Où diable trouver ce maroufle à présent? Mais le voici tout à propos ${ }^{10}$. » L'observation de Valère implique une entrée en scène de Sganarelle qui permettra l'ouverture de la seconde scène. Cet exemple, qui est loin d'être unique, nous montre combien les entrées et sorties structurent la pièce et la parole des personnages.

\section{- Autres déplacements}

Il existe d'autres types de déplacements scéniques dans les comédies de Molière, à savoir des déplacements nécessités par l'action qui se déroule sur scène uniquement. En général, il s'agit de l'ordre donné par un personnage à un autre de quitter la scène. 
Ainsi Arnolphe, dans L'Ecole des femmes, intime-t-il l'ordre de se déplacer à ses domestiques, ou le leur interdit: «Levez-vous et rentrant, faites qu'Agnès descende. / Arrêtez. Sa surprise en deviendrait moins grande ${ }^{11}$. » Dans ce cas, les ordres lancés par Arnolphe sont immédiatement suivis de leur mise en œuvre, ce qui donne lieu à une scène où les déplacements, aussitôt commencés, sont stoppés par un ordre contradictoire. Ainsi, on le voit, la parole des personnages dans les comédies de Molière, est imprégnée des déplacements scéniques : c'est souvent elle qui les motive, les cadre et les limite.

\section{- Localisation du déplacement dans l'espace}

17 Le déplacement d'un personnage dans l'espace scénique dépend de la direction de son mouvement déterminée par un repère : il s'agira de s'avancer vers un objet, ou au contraire, de s'en éloigner. Dans plusieurs cas, un déplacement est localisable scéniquement par rapport à un autre déplacement qui a lieu simultanément sur scène. Dans Le Médecin volant, c'est Sabine qui dit à Gorgibus à propos de Sganarelle : « Le voilà qui me suit ${ }^{12}$ ». Ici, les déplacements scéniques sont tous dépendants d'une donnée sur scène : celui de Sabine est fonction de la localisation de son oncle, puisqu'elle s'avance vers lui, et le déplacement de Sganarelle est orienté selon celui - qu'il doit approximativement calquer - de la jeune fille. Ce type d'exemple n'est pas le seul dans les comédies de Molière. Il met en évidence les rapports qui existent et évoluent spatialement entre les personnages.

\section{- Type de déplacement}

Nous avons peu d'indications implicites pour déterminer les types de déplacements, ou la manière particulière dont un personnage se meut sur scène. Cependant, nous en trouvons une occurrence particulière dans L'Avare. En effet, le personnage de La Flèche y est qualifié par Harpagon de "boiteux ${ }^{13}$ ", ce qui ne nous étonne guère puisque le comédien pour lequel le rôle avait à l'origine été écrit - il s'agit de Louis Béjart - avait lui-même une démarche boiteuse. Autrement dit, le texte tient compte, intrinsèquement, des caractéristiques du comédien. En conséquence, ce qui n'était qu'un fait extérieur à la pièce devient pour L'Avare constitutif du personnage. Il se transcrit ainsi en didascalie implicite fort claire. Ajoutons l'exemple du Bourgeois Gentilhomme, où le personnage prend des cours de danse. Son déplacement scénique abandonne par conséquent le mode de la marche pour lui préférer celui de la chorégraphie. Ainsi, si les indications implicites sur les types de déplacements sont rares, elles sont toujours importantes.

\section{- La vitesse de déplacement}

La vitesse de déplacement est souvent révélatrice de l'intention d'un personnage. Dans ce cas, le texte de Molière n'hésite pas à la suggérer au sein du dialogue, car elle est alors dramaturgiquement signifiante. Ainsi, Elmire fait remarquer à Madame Pernelle : "Vous marchez d'un tel pas qu'on a peine à vous suivre ${ }^{14}$ ». Ici, la vitesse de Madame Pernelle manifeste sa volonté de quitter les lieux, ce qu'explicitera par la suite son règlement de compte avec les membres de sa famille. Autre exemple, autre intention, avec La Jalousie du Barbouillé, où le personnage éponyme expliquera, dans sa volonté de rattraper le médecin qui vient de sortir de scène : «Je m'en vais courir après lui ${ }^{15}$ ». Ces 
exemples, que nous bornerons à ce petit nombre, nous montrent comment Molière joue sur les rythmes de la représentation: les bouleversements de la vitesse de jeu permettent ainsi de conférer à la pièce un tempo qui n'est pas uniforme.

\section{Les grimaces} leurs grimaces. Mais ces dernières ne sont que rarement explicitées par le texte de façon claire : cela fait partie des cas où le texte porte en creux la trace d'un jeu dont il ne définit pas la substance. Dans George Dandin, par exemple, le personnage principal est victime de la raillerie silencieuse de sa femme : «[...] c'est fort mal à vous d'en user comme vous faites. [...] et vous n'avez que faire de hocher la tête, et de me faire la grimace ${ }^{16}$. $»$ Cependant, dans certains cas fort rares le type de grimace est précisé, et Molière n'hésite pas à jouer sur ce phénomène comique. Relevons l'exemple du Bourgeois Gentilhomme, où la grimace est cette fois question de parole: il s'agit de l'extrait où le maître de philosophie apprend à Monsieur Jourdain à prononcer les voyelles. Cela donne lieu à une scène haute en couleur où le bourgeois met à contribution ses lèvres en grimaces qu'on imagine excessives. On sait d'ailleurs combien Molière était réputé pour sa capacité à décomposer son visage et à le soumettre à des expressions comiques. On voit bien, ici, que ses textes en portent la trace jusque dans les dialogues.

\section{Les gestes}

\section{- Les gestes banals}

Les comédies de Molière sont riches en gestes du quotidien, qu'il s'agisse de gestes servant à désigner un objet ou un personnage - le recours aux démonstratifs et déictiques est alors très fréquent - ou bien de gestes de civilité - de l'ordre des usages de la bonne société et de la politesse. Nous pouvons définir les gestes civils comme une mise en mouvement de la parole verbale : les formules civiles, insérées dans le langage dramatique ont en effet, chez Molière, un correspondant gestuel. Dans La Comtesse d'Escarbagnas, notamment, la Comtesse exige: «Comte, saluez Madame. Faites la révérence à Monsieur le Vicomte. Saluez Monsieur le conseiller ${ }^{17}$ ». Le salut est fréquent chez Molière, mais il peut être accompagné de coups de chapeaux, de formules de politesse... Autant de marques qui actualisent les comédies et permettent au lecteur contemporain une meilleure visualisation du jeu de scène, où la gestuelle du quotidien prend une place non négligeable.

\section{- Les gestes signifiants}

Nous remarquons également la présence de gestes signifiants, c'est-à-dire des gestes qui ont une signification dramaturgique et fonctionnent comme une parole du corps. Ils sont constitués d'une part de gestes révélateurs, tels que les signes de tête, les gestes familiers (le bâillement par exemple), les gestes violents (comme les coups de bâton); et d'autre part de gestes symboliques qui ont souvent pour intention de conclure un pacte. C'est le cas lorsque les personnages cherchent à se réconcilier après une dispute. Dans Le Tartuffe, notamment, Dorine tente de réunir Valère et Mariane, qui se sont querellés, en leur ordonnant : "Vous êtes fous tous deux. Çà, la main l'un et l'autre ${ }^{18}$ ». 
Cet exemple est courant. Il s'agit pour les personnages d'engager mutuellement leur foi : le geste de la main scellera cet engagement. On voit ainsi comment les gestes, dans les comédies de Molière, n'ont pas toujours une valeur usuelle, mais peuvent être le symbole scénique d'un engagement personnel invisible sans le recours au geste. Dès lors, le geste, sollicité par la parole, atteint un degré supérieur de signification en gagnant du terrain sur la parole elle-même.

\section{La parole}

La parole symbolise l'incarnation verbale d'un personnage. Chez Molière, son expression scénique est particulièrement travaillée. Il va donc ici s'agir de repérer les indices qui permettent au lecteur de se représenter mentalement la manière dont le texte de théâtre est proféré sur scène par les comédiens.

\section{- Les types de voix}

Bien souvent, les types de voix sont fonction de l'état du personnage qui s'exprime sur scène. Les éclats de voix sont d'ailleurs nombreux. Ainsi, dans Le Tartuffe, Orgon est amené à crier devant sa mère, qui refuse de l'entendre :

Je l'ai vu, dis-je, vu, de mes propres yeux vu,

Ce qu'on appelle vu : faut-il vous le rebattre

Aux oreilles cent fois et crier comme quatre $?^{19}$

Ici, la colère explique son cri. Cependant, hausser le ton, pour un personnage de Molière, peut recouvrir d'autres significations. Il pourra s'agir par exemple d'interpeller un personnage, ou de faire état de son étonnement. Car Molière sait également jouer sur les nuances. Ainsi, la voix peut se moduler à la représentation. On trouve notamment des occurrences implicites du travestissement de voix, comme dans Le Médecin volant, où Sganarelle tente de faire croire à l'existence de son jumeau à Gorgibus. D'une manière générale, dès lors qu'il y a déguisement, il semblerait qu'il y ait travestissement de la voix. Il peut également arriver que Molière attribue par essence un type de voix à certains personnages, comme c'est le cas pour Climène qui "affecte toujours un ton de voix languissant et niais ${ }^{20}$ ", ou pour le personnage du poète qui adopte un ton "sentencieux ${ }^{21}$ » et pédant. La voix, dans les pièces de Molière, est donc un facteur d'animation scénique, qui joue sur les modes de représentation et la diversité du jeu.

\section{- Les intonations}

L'intonation recouvre « l'ensemble des éléments prosodiques qui expriment l'émotivité du locuteur ${ }^{22}$ » mais également l'inflexion de voix qui s'adapte à la structure syntaxique de la phrase, et à sa ponctuation. On ne peut donc déterminer avec précision l'intonation dans les comédies de Molière. On remarquera tout de même combien Molière se plaît à varier les intonations au sein d'une même réplique. Outre le jeu sur la ponctuation - sur lequel nous ne nous attarderons pas étant donné le peu de fiabilité avec laquelle elle nous a été transmise - des procédés comme l'incise se situent au cœur de cette démarche qui vise à rompre l'intonation parfois trop uniforme d'une parole. Les nombreuses exclamations et interjections ont ce même rôle de rupture. Dans l'exemple suivant, où Done Elvire s'exclame : « Hé bien! Sganarelle, parlez. Il n'importe 
de quelle bouche j'entende ces raisons ${ }^{23}$ ", l'exclamation pose la tonalité de départ, et constitue une accroche à partir de laquelle la tonalité du reste de la phrase est dynamisée. Ces modèles de phrase sont fréquents chez Molière, et demeurent pour nous des indices privilégiés. Parfois au contraire, l'uniformité d'intonation au sein d'une réplique nous frappe, dans le cas notamment d'accumulations de phrases du même mode - de type interrogatives ou exclamatives. Mais cette uniformisation de la réplique, visant à l'expressivité de la parole, accentuera d'autant le sentiment du locuteur.

\section{- Le tempo}

Le tempo se définissant comme la vitesse de diction d'une réplique, d'un enchaînement de répliques ou d'une scène toute entière, il nous apparaît difficile de le déterminer avec précision dans les textes de Molière. La vitesse d'enchaînement des répliques constitue un des moyens privilégiés de variation du tempo. On le constate notamment dans les cas - courants - où plusieurs interventions de personnages se concentrent sur un alexandrin. La notion de silence, dès lors qu'il s'agit du tempo, devient centrale. Elle est manifeste dans le cas des répliques dont l'inachèvement interrompt temporairement la diction, ce qui n'est pas rare chez Molière. De plus, l'ampleur d'une réplique tend à ralentir l'élocution des personnages, contrairement aux répliques brèves ou scandées qui l'accélèrent, ainsi que dans celle d'Oronte qui s'exclame : « Ah ! j'ai tort, je l'avoue, et je quitte la place./Je suis votre valet, Monsieur, de tout mon cœur ${ }^{24}$ ». Le tempo général peut être aussi altéré par la structure des répliques, notamment grâce à la ponctuation, au mode (interrogatif, exclamatif...) des phrases, ou encore au rythme.

\section{- Le rythme}

Le rythme d'un discours constitue la cadence, régulière ou irrégulière, imprimée par la distribution d'éléments linguistiques, tels que les accentuations, répétitions... Il s'agit donc d'observer la façon dont le texte se respire. On note chez Molière un souci de rythmer la parole des personnages où, souvent, la répétition des mêmes éléments verbaux est créatrice de rythme. Il en est ainsi lorsqu'Harpagon crie au vol: "Au voleur! Au voleur! à l'assassin! au meurtrier ${ }^{25}$ ! D'autres procédés tels que l'incise, sur lesquels nous ne reviendrons pas car ils sont communs au rythme et au tempo, sont des facteurs indiscutables de rythme. Ils bouleversent la respiration classique de la phrase, en imposant au comédien une ou plusieurs pauses durant la diction. Certains discours particulièrement longs sont ainsi rythmés par le dramaturge. Par ailleurs, le vers est naturellement plus rythmé que la prose. Mais Molière y ajoute des effets, en jouant sur les structures qu'il permet. Ainsi, et pour exemple, les jeux de symétrie sont fréquents, qui rythment la réplique de manière parfaitement maitrisée. A cela s'ajoutent d'autres moyens tels que l'antéposition, qui entraîne une accentuation toute différente du vers. Autrement dit, de nombreux procédés permettent à Molière de rythmer sa phrase : autant d'indices que le lecteur doit savoir écouter.

Cette typologie, qui n'a pu se permettre d'être exhaustive, nous montre néanmoins combien la parole moliéresque intègre le jeu des comédiens. Jeu et parole ne sont plus, comme le théorise l'abbé d'Aubignac dans sa Pratique du théâtre, deux éléments distincts mais demeurent étroitement liés ${ }^{26}$. D'ailleurs, on remarquera combien Molière a su 
dépasser cette théorie de ce que nous nommons aujourd'hui didascalie implicite, pour se l'approprier. Ce qui n'était qu'esquissé dans l'esprit du théoricien devient un enjeu majeur de fonctionnement et d'efficacité des pièces de Molière. La didascalie implicite n'est plus seulement question de mise en scène, elle devient un révélateur psychologique, un facteur de cohérence du texte. Molière a compris qu'elle pouvait offrir de nouvelles ressources au texte de théâtre. Elle n'est pas seulement, chez lui, un moyen en vue d'une fin - celle de la mise en scène du texte - mais bien une fin en soi puisqu'elle révèle le personnage qui y recourt. Ainsi, parmi d'autres, l'exemple de la critique par Sganarelle du costume de son frère, Ariste, nous montre à quel point l'indication implicite permet au dramaturge de révéler la psychologie du personnage. Sganarelle nous y apparaît comme un personnage renfermé et porteur de valeurs rétrogrades. D'un point de vue dramaturgique, cette description oriente la représentation de façon binaire: aux deux états d'esprit des personnages correspondent deux costumes parfaitement opposés, l'un étant révélateur de l'autre. L'expressivité des costumes vient ici parachever l'efficacité de la pièce. On voit bien comment Molière réussit à mêler ici ce qui est de l'ordre du descriptif nécessaire à la mise en scène et ce qui est de l'ordre de l'axiologique. Il fait donc évoluer le dialogue vers un approfondissement de sa propre dramaturgie. Nous irons même plus loin: grâce à la didascalie implicite, le geste, chez Molière, peut se faire parole. Et c'est précisément parce qu'en homme de scène, Molière en a percé les ressources et exploré les enjeux, que la didascalie implicite influence le texte de théâtre d'une façon bien plus importante chez lui qu'elle ne semblait devoir le faire dans la théorie de d'Aubignac. Avec Molière, les répercussions des didascalies implicites dans le texte de comédie sont donc toutes nouvelles.

\section{NOTES}

1. Molière, Avis au lecteur de L'Amour médecin, Euvres Complètes, tome 2, Paris, Gallimard, «Bibliothèque de la Pléiade ", 1971, p. 95.

2. Anne Ubersfeld, Les Termes clés de l'analyse du théâtre, Paris, Dunod, 1996, p. 29.

3. Molière, L'Ecole des femmes, op. cit., acte I, sc.4, v 317-318, p. 561.

4. Molière, L'Ecole des maris, op. cit., acte I, sc.1, v 23-40, p. 418.

5. Molière, Le Misanthrope, op. cit., acte V, sc IV, p 213.

6. Sabine Chaouche, L'Art du comédien. Déclamation et jeu scénique en France à l'âge classique (1629-1680), Genève, Honoré Champion, 2001, p. 172.

7. Molière, Le Bourgeois Gentilhomme, op. cit., acte II, sc.1, p. 721.

8. 1 : Molière, L'Ecole des Femmes, op. cit., acte III, sc.1, v 665, p. 579; 2 : La Comtesse d'Escarbagnas, op. cit., sc.2, p. 958; 3 : Le Malade Imaginaire, op. cit., acte II, sc.5, p. 1133.

9. Molière, Les Précieuses ridicules, op. cit., sc.9, p. 279-280.

10. Molière, Le Médecin volant, op. cit., sc.1, p. 32.

11. Molière, L'Ecole des femmes, op. cit., acte II, sc.2, p. 565, v 411-412.

12. Molière, Le Médecin volant, op. cit., sc.4, p. 34.

13. Molière, L'Avare, op. cit., acte I, sc.3, p. 523. 
14. Molière, Le Tartuffe, op. cit., acte I, sc.1, v 2.

15. Molière, La Jalousie du Barbouillé, op. cit., sc.2, p. 16.

16. Molière, George Dandin, op. cit., acte II, sc.2, p. 481.

17. Molière, La Comtesse d'Escarbagnas, op. cit., acte I, sc.7, p. 967.

18. Molière, Le Tartuffe, op. cit. p. 934, v 781-782.

19. Molière, Le Tartuffe, op. cit., acte V, sc.3, p. 972, v 1676-1678.

20. Molière, La Critique de L'Ecole des femmes, op. cit., sc.2, p. 645.

21. Molière, L'Impromptu de Versailles, op. cit., p. 682.

22. Gabriel Conesa, Le Dialogue moliéresque, étude stylistique et dramaturgique, Paris, PUF, 1983, p. 351.

23. Molière, Dom Juan, op. cit., p. 39.

24. Molière, Le Misanthrope, op. cit., acte I, sc.2, p. 160, v 436-437.

25. Molière, L'Avare, op. cit., acte IV, sc.7, p. 569.

26. Pour d'Aubignac, en effet, les indications de l'ordre de la mise en scène n'ont pas à figurer dans le texte, car elles y introduisent une hétérogénéité en accordant une place à la voix de l'auteur - à l'instar du « poème épique ». De fait, d'Aubignac ne conçoit pas que les didascalies puissent servir le texte dramatique et son efficacité. Dans son chapitre intitulé «De quelle manière le poète doit faire connaître la décoration et les Actions nécessaires dans une Pièce de Théâtre ", le théoricien affirme que dans une bonne pièce, on devrait pouvoir, sans aucune indication, deviner « [...] le nom, la qualité, les habits, l'équipage, les gestes, les intérêts de tous ceux qui parlent, ce que chacun doit dire, le lieu de la Scène et ses décorations, l'étendue du Poème et tout ce qui doit faire partie de l'Action Théâtrale : tant il est vrai que dans une pièce régulière tout $\mathrm{y}$ doit être aussi facilement connu de l'Esprit, que des yeux. Et tout Poème Dramatique qui ne pourra se faire connaître de la sorte est assurément défectueux. » La Pratique du théâtre, Abbé François Hédelin d'Aubignac, Genève, Slatkine Reprints, 1996, chap VIII, p 55. C'est à cette excessive rigueur que Molière répond, de manière directe ou non, par son propre usage bien plus souple de l'indication scénique, qui n'hésite pas, malgré tout, si nécessaire, à faire appel à des didascalies explicites, et surtout, qui dépasse cette conception purement utilitaire (du point de vue de la mise en scène) de ce que nous appelons didascalie implicite. 